\title{
Belphégor
}

\section{José Manuel Trabado, dir. Encrucijadas gráfico- narrativas. Novela gráfica y álbum ilustrado}

\section{Jan Baetens}

\section{(2) OpenEdition}

1 Journals

\section{Édition électronique}

URL : https://journals.openedition.org/belphegor/3989

DOI : 10.4000/belphegor.3989

ISSN : 1499-7185

Éditeur

LPCM

\section{Référence électronique}

Jan Baetens, « José Manuel Trabado, dir. Encrucijadas gráfico-narrativas. Novela gráfica y álbum ilustrado », Belphégor [En ligne], 19-1 | 2021, mis en ligne le 24 juin 2021, consulté le 06 juillet 2021. URL : http://journals.openedition.org/belphegor/3989; DOI : https://doi.org/10.4000/belphegor.3989

Ce document a été généré automatiquement le 6 juillet 2021.

\section{(c) (i) (9)}

Belphégor est mis à disposition selon les termes de la Licence Creative Commons Attribution - Pas d'Utilisation Commerciale - Pas de Modification 4.0 International. 


\section{José Manuel Trabado, dir. Encrucijadas gráfico-narrativas. Novela gráfica y álbum ilustrado}

Jan Baetens

\section{RÉFÉRENCE}

José Manuel Trabado, dir. Encrucijadas gráfico-narrativas. Novela gráfica y álbum ilustrado. Somonte-Gijón : éd. TREA. 2020, 320 p., ISBN: 978-84-18105-08-1 


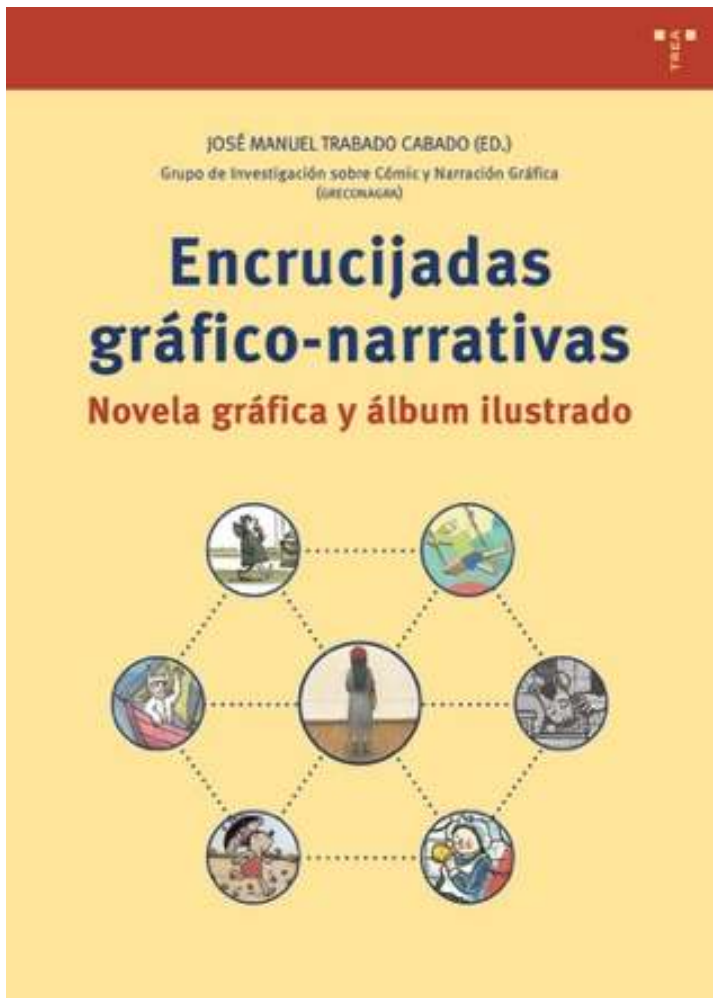

1 Encrucijadas gráfico-narrativas. Novela gráfica y álbum ilustrado ("Carrefours graphiconarratifs. Roman graphique et album illustré") est une publication collective émanant du groupe «Greconagra », que dirige à l'Université de Léon le professeur José Manuel Trabado. Il s'inscrit en marge de la collection Grafikalismos, issue des mêmes chercheurs mais publiée par les Presses Universitaires de Léon. ${ }^{1}$ Il témoigne du dynamisme des recherches sur la narration graphique en langue espagnole et de l'important appui institutionnel d'une maison soucieuse de la qualité formelle de ses ouvrages (format généreux, papier de bon grammage, mise en page soignée, iconographie d'une richesse vraiment exceptionnelle - dans le volume en question, pas moins de 99 illustrations en couleur, presque un livre dans le livre).

2 L'essentiel, toutefois, demeure le contenu et sur ce point les livres de la collection comportent un double avantage. Tout en s'inscrivant dans les grands axes de la recherche contemporaine, continentale aussi bien qu'anglo-saxonne, la plupart des études, certaines monographiques, d'autres collectives, donnent d'abord accès à la production hispanophone, encore trop peu connue en Europe. Mais elles abordent aussi des questions originales qui dépassent de loin le cadre de la seule péninsule.

Encrucijadas est un parfait exemple de pareil double programme. D'une part, le livre combine deux corpus : national et international. D'autre part, il remet sur le devant de la scène un problème capital que les recherches plus récentes avaient eu tendance à laisser un peu de côté, à savoir la rencontre, mais non la fusion, de la bande dessinée et de l'album illustré, plus exactement du picturebook, genre et médium davantage ancrés dans la culture anglaise et américaine que dans d'autres aires et traditions. La raison principale de ce relatif oubli n'est cependant pas seulement linguistique. De manière plus fondamentale, comme le rappelle avec justesse José Manuel Trabado dans son excellente présentation du volume, cette omission tient avant tout aux métamorphoses de la bande dessinée depuis plusieurs décennies. Plusieurs éléments méritent ici d'être 
mis en avant. Pour commencer, le clivage du picturebook, qui s'adresse toujours et toujours simultanément à deux publics (d'un côté les enfants, qui lisent et regardent, de l'autre les adultes, qui surveillent et accompagnent cette lecture - sans oublier que c'est toujours eux qui décident de l'achat) et de la bande dessinée, qui se lit (toujours) et s'achète (parfois) hors tout contrôle parental. Ensuite, et pas seulement en Espagne, l'érosion de la bande dessinée pour enfants, remplacée par d'autres types de divertissement comme les jeux vidéo. Enfin, l'hégémonie croissante d'une forme de bande dessinée, le roman graphique, qui se veut aux antipodes du caractère supposé didactique ou moralisateur de l'album illustré. En même temps, la bande dessinée moderne a perdu depuis bien du temps son homogénéité matérielle et institutionnelle. Elle a évolué dans des directions très diverses, dont certaines la rapprochent incontestablement du picturebook, surtout dans ses formes résolument «adultes». De ces transformations il a résulté non seulement une série d'échanges et de chevauchements, la frontière entre bande dessinée ou roman graphique et album illustré devenant de plus en plus floue, mais aussi une interrogation plus fondamentale: peut-on encore distinguer les deux, et si oui, comment et surtout pourquoi?

4 Voilà les grandes questions au cœur de ce livre, qui les traite d'un grand nombre de points de vue : sémiotique, historique, narratologique, politique, esthétique, didactique. De cet ensemble se dégagent trois grandes lignes de force.

5 Premièrement, toutes les contributions attachent une place essentielle au support matériel des œuvres, en général (le choix entre magazine et livre, par exemple) comme en particulier (format, couleur, mise en pages, etc.). Cette orientation sur la base matérielle des productions aide Encrucijadas à positionner l'étude de la narration graphique au sein de l'histoire culturelle du livre, un élargissement que l'on observe aussi en d'autres publications récentes comme celles, en France, de Sylvain Lesage, Julien Baudry ou encore Nicolas Labarre.

6 En second lieu, Encrucijadas est aussi un livre qui joue de manière intelligente avec le problème épineux des genres. Face à des œuvres inclassables ou résolument hybrides, comme par exemple celles de Marion Fayolle, Sergio García ou le tandem Dave McKean et Neil Gaiman, il est toujours tentant de postuler d'emblée que la question du genre perd de sa pertinence et force est en effet de constater que le souci d'éviter les étiquettes traverse l'ensemble des chapitres du livre. Cependant, il serait erroné de croire que la narration graphique, qui dans la très grande majorité des cas fonctionne au sein de la littérature de grande diffusion, ne s'appuie plus sur des indications génériques, à la fois repères et tremplins. L'existence d'un cadre et d'une grille génériques est un outil indispensable, dont se sert n'importe qui se frotte à la littérature populaire. Ignorer cet horizon serait une piètre erreur et c'est un plaisir de noter que les contributeurs du livre, sans tomber dans le piège des débats un rien stériles entre tenants de bande dessinée et défenseurs de roman graphique ou entre littérature enfantine et production pour adultes, tiennent compte des réalités génériques pour examiner avec soin la manière dont les créateurs reprennent, déjouent, transforment les règles des genres sans jamais en sous-estimer l'intérêt.

7 Troisièmement, enfin, Encrucijadas est aussi un livre qui ne renonce pas aux plaisirs et aux mérites de la microscopie. Les divers chapitres, qui tous partent des mêmes questionnements théoriques, n'en restent jamais aux réflexions abstraites mais se donnent le temps de lire les mots et les images dans le texte, c'est-à-dire sur la page, 
dans le livre et au-delà. En effet, chaque analyse inclut et commente aussi la réception des œuvres, soit qu'on se penche sur les problèmes ayant généré telle ou telle pratique (comme c'est le cas de la belle analyse des Classic Children's Comics, la tentative d'Art Spiegelman et Françoise Mouly de réinventer la littérature enfantine dans le médium bande dessinée au moment même où la bande dessinée pour enfants paraissait en voie d'épuisement), soit que l'accent se porte sur la réception des œuvres et leurs effets tantôt voulus, tantôt inattendus (l'exemple de Raymond Briggs, porté aux nues pour la teneur politique de son travail mais ignoré en tant que précurseur de la bande dessinée autobiographique, est là pour montrer combien l'accueil et la postérité des créations demeurent souvent imprévisibles).

Livre-carrefour, comme son titre l'affiche, Encrucijadas présente une belle dialectique de deux forces antagonistes: divergence et convergence. C'est une livre qui force l'étude de la bande dessinée à explorer de nouvelles pistes -et notamment à jeter des ponts vers l'analyse du picturebook, de l'album illustré ou de la poésie graphique. Mais c'est aussi un livre qui ne perd pas de vue l'importance des objets et de leur inscription dans des ensembles éditoriaux, commerciaux, culturels et politiques plus vaste.

\section{NOTES}

1. Pour plus de détails, voir: https://servicios.unileon.es/publicaciones/grafikalismos/

\section{INDEX}

Mots-clés : roman graphique, Espagne, album illustré

Palabras claves : Novela grafica, Album ilustrado, José Manuel Trabado

\section{AUTEUR}

\section{JAN BAETENS}

Jan Baetens est professeur d'études culturelles à l'université de Leuven, où il s'intéresse notamment aux rapports entre texte et image dans des genres hybrides tels que la bande dessinée ou le roman-photo. Quelques publications récentes sont: Adaptation et bande dessinée (Les Impressions Nouvelles, 2020) et Une fille comme toi (JBE éditions, 2020), un "remix" d'une collection de ciné-romans-photos à partir du scénario imaginaire d'un film typique de la Nouvelle Vague.

jan.baetens@kuleuven.be 